\title{
Delay-Dependent Robust Exponential Stability for Uncertain Neutral Stochastic Systems with Interval Time-Varying Delay
}

\author{
Weihua Mao, ${ }^{1,2}$ Feiqi Deng, ${ }^{1}$ and Anhua Wan ${ }^{3}$ \\ ${ }^{1}$ College of Automation Science and Engineering, South China University of Technology, \\ Guangzhou 510640, China \\ ${ }^{2}$ Department of Mathematics, South China Agricultural University, Guangzhou 510642, China \\ ${ }^{3}$ School of Mathematics and Computational Science, Sun Yat-Sen University, Guangzhou 510275, China \\ Correspondence should be addressed to Anhua Wan, wananhua@mail.sysu.edu.cn
}

Received 25 April 2012; Revised 27 July 2012; Accepted 30 July 2012

Academic Editor: Jitao Sun

Copyright (C) 2012 Weihua Mao et al. This is an open access article distributed under the Creative Commons Attribution License, which permits unrestricted use, distribution, and reproduction in any medium, provided the original work is properly cited.

\begin{abstract}
This paper discusses the mean-square exponential stability of uncertain neutral linear stochastic systems with interval time-varying delays. A new augmented Lyapunov-Krasovskii functional (LKF) has been constructed to derive improved delay-dependent robust mean-square exponential stability criteria, which are forms of linear matrix inequalities (LMIs). By free-weight matrices method, the usual restriction that the stability conditions only bear slow-varying derivative of the delay is removed. Finally, numerical examples are provided to illustrate the effectiveness of the proposed method.
\end{abstract}

\section{Introduction}

Dynamical systems, such as the distributed networks, electric power systems, and communication systems, can be efficiently modeled by neutral stochastic functional differential equations, which have been extensively studied in recent years, see [1-8] and references therein. Basically, sufficient conditions on stability of time delay systems are divided into two categories: delay-dependent and delay-independent cases. It is well known that the latter is less conservative than the former, especially when the delay is very small. Therefore, increasing attention has been focused on delay-dependent stability analysis of delay systems in recent years, see [1-25].

There are unstable systems without delay that can be stabilized with proper nonzero delay, see [26]. Therefore, it is of great significance to consider the stability of systems with interval time-varying delay, it is a time delay that varies in an interval, in which the lower bound is not restricted to 0 . Recently, interval time-varying delay was introduced and investigated, see [11-17]. 
In the past years, as an effective approach of improving the performance of delaydependent stability criteria, free-weighting matrices method has attracted much attention, see $[2-5,10-12]$. The mean-square exponential stability was studied for neutral stochastic systems with fixed delays in $[2,3]$. The global asymptotic stability for a class of neutral stochastic neural networks was studied in [4]. Improved delay-dependent robust stability criteria of uncertain stochastic systems with interval time-varying delay were proposed in [12].

The usual restriction on derivative of the time-varying delay that $\dot{\tau}(t)<1$ in $[5-7,18]$ brings conservatism, which shows that the resulting conditions can only bear slow-varying delay. In order to remove this limitation, fast-varying rate condition was studied in $[4,12,23]$.

Stability of stochastic differential delay systems with nonlinear impulsive effects is studied in $[24,25]$, the equivalent relations are established between the stability of the stochastic differential delay equations with impulsive effects and that of a corresponding stochastic differential delay equations without impulses.

To our best knowledge, few works on the robust delay-dependent exponential stability analysis have been reported for uncertain neutral stochastic distributed system with fast-varying interval time-varying delay. This paper focuses on the stability analysis of uncertain neutral stochastic distributed system with interval time-varying delay. By Lyapunov-Krasovskii functional theory and free-weighting matrices method, a new delaydependent mean-square exponential stability criterion is formulated in terms of LMI, and the usual restriction of $\dot{\tau}(t)<1$ is removed. Finally, three numerical example are given to illustrate the effectiveness of the proposed method.

Notation 1. Throughout this paper, the notations are standard. If $A$ is a vector or matrix, its transpose is denoted by $A^{T} . P>0$ means that $P$ is a symmetric positive definite matrix. $*$ denotes the symmetry part of a symmetry matrix. $\star$ indicates terms that can be induced by symmetry, for example, $A+A^{T}=A+\star$ and $M P M^{T}=M P \star$. Denote by $\lambda_{M}\{\cdot\}$ and $\lambda_{m}\{\cdot\}$ the maximum and minimum eigenvalue of a matrix, respectively. Let $\rho(\cdot)$ denote the spectral norm of a matrix, while $|\cdot|$ refers to the Euclidean vector norm. Let $(\Omega, \mathcal{F}, P)$ be a complete probability space relative to an increasing family $\{\mathscr{F}\}_{t \geq 0}$ of $\sigma$ algebras $\mathcal{F}_{t} \subset \mathcal{F}$ and $\mathbb{E}\{\cdot\}$ the mathematical expectation operator with respect to the probability measure $p$. $\mathcal{L}_{2}[0, \infty)$ denotes the space of square integrable vector functions over $[0, \infty)$. Let $\tau>0$ and $C\left([-\tau, 0], \mathrm{R}^{n}\right)$ denote the family of all continuous $\mathrm{R}^{n}$-valued functions $\phi$ on $[-\tau, 0]$ with the norm $\|\phi\|=\sup \{|\phi(\theta)|:-\tau \leqslant \theta \leq 0\}$. Let $\mathcal{L}_{\mathcal{F}_{0}}^{2}\left([-\tau, 0] ; \mathrm{R}^{n}\right)$ be the family of all $\mathcal{F}_{0}$-measurable bounded $C\left([-\tau, 0], \mathrm{R}^{n}\right)$-valued random variables $\varphi=\{\varphi(\theta):-\tau \leqslant \theta \leqslant 0\}$.

\section{Preliminaries}

Consider the following uncertain Itô-type neutral stochastic system with both discrete and distributed interval time-varying delays

$$
\begin{gathered}
(\Sigma): \mathrm{d}\left[x(t)-D x\left(t_{\tau}\right)\right] \\
=\left\{\left[A_{0}+\Delta A_{0}(t)\right] x(t)+\left[A_{1}+\Delta A_{1}(t)\right] x\left(t_{\tau}\right)+\left[A_{2}+\Delta A_{2}(t)\right] \int_{t_{\tau}}^{t} x(s) \mathrm{d} s\right\} \\
+\left\{\left[B_{0}+\Delta B_{0}(t)\right] x(t)+\left[B_{1}+\Delta B_{1}(t)\right] x\left(t_{\tau}\right)+\left[B_{2}+\Delta B_{2}(t)\right] \int_{t_{\tau}}^{t} x(s) \mathrm{d} s\right\} \mathrm{d} B(t), \\
x(t)=\phi(t), \quad t \in\left[-\tau_{2}, 0\right],
\end{gathered}
$$


where $x(t) \in \mathrm{R}^{n}$ is the state vector; $B(t)$ is a standard scalar Brownian motion defined on a complete probability space $(\Omega, \mp, D)$, we assume $\mathbb{E}\{\mathrm{d} B(t)\}=0, \mathbb{E}\left\{\mathrm{d}^{2} B(t)\right\}=\mathrm{d} t . \phi(t)$ is any given initial data in $\mathcal{L}_{q_{0}}^{2}\left([-\tau, 0] ; \mathrm{R}^{n}\right)$. Denote $\eta(t)=x(t)-D x\left(t_{\tau}\right), t_{\tau}=t-\tau(t), t_{\tau_{1}}=t-\tau_{1}, t_{\tau_{2}}=$ $t-\tau_{2}, \tau(t)$ is time-varying delays, satisfying

$$
\dot{\tau}(t) \leqslant \bar{\tau}<+\infty, \quad 0 \leqslant \tau_{1} \leqslant \tau(t) \leqslant \tau_{2}<+\infty, \quad \delta=\tau_{2}-\tau_{1} .
$$

Remark 2.1. It should be noted that system (2.1)-(2.2) encompasses many state space models of neutral delay systems, which can be used to represent many important physical systems such as a large class of distributed networks containing lossless transmission lines, population ecology, heat exchangers, wind tunnel, and water resources systems (see [2729] and the references therein). For example, signal transformation cannot be finished in time due to the finite signal propagation speed in distributed networks containing lossless transmission lines, or to the finite switching speed of amplifiers in electronic networks, so the models only depending on the discrete time delays are not complete, the more exact models should include the distributed time delays, and the delays are found both in the states and in the derivatives of the states.

Remark 2.2. It is worth pointing out that, when a time-varying delay appears, it is usually assumed that $\dot{\tau}(t)<1$ is satisfied and the lower bound of the delay is restricted to be zero in the literature $[5,7,8]$ and so forth. However, in this paper, we only require $\dot{\tau}(t) \leqslant \bar{\tau}$, in addition, the range of delay may vary in a range for which the lower bound is not restricted to be zero. Therefore, the time-varying delay in this paper is more general.

$A_{i}, B_{j}, i, j=0,1,2$, are known real constant matrices of appropriate dimensions; $\Delta A_{i}, \Delta B_{j}, i, j=0,1,2$, are unknown matrices representing time-varying parameter uncertainties. For the sake of convenience, we assume that the uncertainties are norm-bounded and can be described as

$$
\left(\Delta A_{i}(t) \quad \Delta B_{i}(t)\right)=G F(t)\left(S_{i} T_{i}\right)
$$

where $S_{i}, T_{i}, i=0,1,2$, are known real constant matrices, $F(t)$ is an unknown real timevarying function with appropriate dimension satisfying

$$
F^{\mathrm{T}}(t) F(t) \leqslant I
$$

It is assumed that the elements of $F(t)$ are Lebesgue measurable. Throughout this paper, the following assumption, definitions, and lemmas will be used to develop our results.

Assumption 2.3. The difference operator matrix $D$ satisfies $\|D\|<1$.

Definition 2.4 (see [1]). The neutral stochastic delay system (2.1)-(2.2) is said to be meansquare exponentially stable if there is a pair of positive constants $\alpha, \beta$ such that

$$
\mathbb{E}\left\{x^{\mathrm{T}}(t) x(t)\right\} \leqslant \alpha \mathrm{e}^{-\beta t} \sup _{-\tau_{2} \leqslant \theta \leqslant 0} \mathbb{E}\{\bar{\phi}(\theta)\} .
$$


Lemma 2.5 (see [30]). If there exists a vector function $v(t):[0, r] \rightarrow \mathrm{R}^{n}$ such that $\int_{0}^{r} v^{\mathrm{T}}(s) P v(s) \mathrm{d} s$ and $\int_{0}^{r} v(s) \mathrm{d} s$ are well defined, then the following inequality:

$$
\left[\int_{0}^{r} v(s) \mathrm{d} s\right]^{\mathrm{T}} P\left[\int_{0}^{r} v(s) \mathrm{d} s\right] \leqslant r \int_{0}^{r} v^{\mathrm{T}}(s) P v(s) \mathrm{d} s
$$

holds for any pair of symmetric positive definite matrix $P \in \mathrm{R}^{n \times n}$ and $r>0$.

Lemma 2.6 (see [31]). For any vectors $x, y \in \mathrm{R}^{n}$, matrices $A, D, E, P$, and $F$ are real matrices of appropriate dimensions with $P>0$ and $F^{T} F \leqslant I$, the following inequalities hold:

(1) $2 x^{\mathrm{T}} D F E y \leqslant \epsilon^{-1} x^{\mathrm{T}} D D^{\mathrm{T}} x+\epsilon y^{\mathrm{T}} E E^{\mathrm{T}} y$;

(2) for any scalar $\epsilon>0$ such that $P-\epsilon D D^{\mathrm{T}}>0$, then $(A+D F E)^{\mathrm{T}} P^{-1}(A+D F E) \leqslant$ $\epsilon^{-1} E^{\mathrm{T}} E+A^{\mathrm{T}}\left(P-\epsilon D D^{\mathrm{T}}\right)^{-1} A$;

(3) $2 x^{\mathrm{T}} y \leqslant x^{\mathrm{T}} P^{-1} x+y^{\mathrm{T}} P y$.

\section{Main Results}

In this section, a robustly stochastically exponentially stable criterion for the uncertain linear neutral stochastic distributed delayed system $(\Sigma)$ will be established by applying the Lyapunov-Krasovskii theory and free-weighting matrices method.

For convenience, define a new state variable

$$
f(t)=\left(A_{0}+\Delta A_{0}(t)\right) x(t)+\left(A_{1}+\Delta A_{1}(t)\right) x\left(t_{\tau}\right)+\left(A_{2}+\Delta A_{2}(t)\right) \int_{t_{\tau}}^{t} x(s) \mathrm{d} s
$$

and a new perturbation variable

$$
g(t)=\left(B_{0}+\Delta B_{0}(t)\right) x(t)+\left(B_{1}+\Delta B_{1}(t)\right) x\left(t_{\tau}\right)+\left(B_{2}+\Delta B_{2}(t)\right) \int_{t_{\tau}}^{t} x(s) \mathrm{d} s .
$$

Then, system (2.1) becomes

$$
\mathrm{d}\left[x(t)-D x\left(t_{\tau}\right)\right]=f(t) \mathrm{d} t+g(t) \mathrm{d} B(t) .
$$


Applying Leibniz-Newton formula to (2.1), it yields the following zero equations which will be used in our main result:

$$
\begin{gathered}
2 \xi^{\mathrm{T}}(t) N\left[x(t)-D x\left(t_{\tau}\right)-x\left(t_{\tau}\right)+D x\left(t_{\tau}-\tau\left(t_{\tau}\right)\right)-\int_{t_{\tau}}^{t} f(s) \mathrm{d} s-\int_{t_{\tau}}^{t} g(s) \mathrm{d} B(s)\right]=0, \\
2 \xi^{\mathrm{T}}(t) H\left[x\left(t_{\tau_{1}}\right)-D x\left(t_{\tau_{1}}-\tau\left(t_{\tau_{1}}\right)\right)-x\left(t_{\tau}\right)+D x\left(t_{\tau}-\tau\left(t_{\tau}\right)\right)-\int_{t_{\tau}}^{t_{\tau_{1}}} f(s) \mathrm{d} s-\int_{t_{\tau}}^{t_{\tau_{1}}} g(s) \mathrm{d} B(s)\right]=0, \\
2 \xi^{\mathrm{T}}(t) M\left[x\left(t_{\tau}\right)-D x\left(t_{\tau}-\tau\left(t_{\tau}\right)\right)-x\left(t_{\tau_{2}}\right)+D x\left(t_{\tau_{2}}-\tau\left(t_{\tau_{2}}\right)\right)-\int_{t_{\tau_{2}}}^{t_{\tau}} f(s) \mathrm{d} s-\int_{t_{\tau_{2}}}^{t_{\tau}} g(s) \mathrm{d} B(s)\right]=0,
\end{gathered}
$$

where $N, H$, and $M$ are any matrices with appropriate dimensions, and

$$
\begin{aligned}
\xi^{\mathrm{T}}(t)=\left[x^{\mathrm{T}}(t), x^{\mathrm{T}}\left(t_{\tau}\right), \frac{1}{\tau_{2}} \int_{t_{\tau}}^{t} x^{\mathrm{T}}(s) \mathrm{d} s, x^{\mathrm{T}}\left(t_{\tau_{1}}\right), x^{\mathrm{T}}\left(t_{\tau_{2}}\right),\right. \\
\left.x^{\mathrm{T}}\left(t_{\tau_{1}}-\tau\left(t_{\tau_{1}}\right)\right), x^{\mathrm{T}}\left(t_{\tau_{2}}-\tau\left(t_{\tau_{2}}\right)\right), x^{\mathrm{T}}\left(t_{\tau}-\tau\left(t_{\tau}\right)\right)\right] .
\end{aligned}
$$

With zero equations (3.4) above, we obtain the following theorem.

Theorem 3.1. For given scalars $0 \leqslant \tau_{1} \leqslant \tau_{2}$ and $\bar{\tau}$, system $(\Sigma)$ is robustly stochastically exponentially stable for all time-varying delays satisfying (2.3) and for all admissible uncertainties satisfying (2.4) and (2.5), if there exist symmetric positive definite matrices $P>0, R_{i}>0, i=$ $0,1,2, \ldots, 10$, scalars $\epsilon_{j}, j=1,2,3$ and any matrices $N, H, M$ with appropriate dimensions, such that the following LMI (3.6) holds:

$$
\Phi=\left(\begin{array}{cc}
\Phi_{1} & \Phi_{2} \\
* & \Phi_{3}
\end{array}\right)<0
$$

where

$$
\Phi_{1}=\left(\begin{array}{cccccc}
\Theta & \bar{P} G & V_{1}^{T} Z & 0 & V_{2}^{T} U & 0 \\
* & -\epsilon_{1} I & 0 & 0 & 0 & 0 \\
* & * & -Z & Z G & 0 & 0 \\
* & * & * & -\epsilon_{2} I & 0 & 0 \\
* & * & * & * & -U & U G \\
* & * & * & * & * & -\epsilon_{3} I
\end{array}\right)
$$




$$
\begin{aligned}
& \Phi_{2}=(\bar{N} \bar{H} \bar{M} \bar{N} \bar{H} \bar{M}),
\end{aligned}
$$

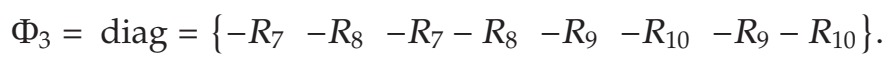

$$
\begin{aligned}
& \Theta=\Gamma+\Psi+\Psi^{\mathrm{T}}, \quad V_{1}=\left[\begin{array}{llllllll}
A_{0} & A_{1} & A_{2} & 0 & 0 & 0 & 0 & 0
\end{array}\right], \quad V_{2}=\left[\begin{array}{llllllll}
B_{0} & B_{1} & B_{2} & 0 & 0 & 0 & 0 & 0
\end{array}\right], \\
& \bar{P}=\left[\begin{array}{llllllll}
P & -P D & 0 & 0 & 0 & 0 & 0 & 0
\end{array}\right]^{\mathrm{T}}, \quad Z=\tau_{2} R_{7}+\delta R_{8}, \quad U=P+\tau_{2} R_{9}+\delta R_{10}, \\
& \bar{N}=\left[N^{\mathrm{T}}, 0,0,0,0,0\right]^{\mathrm{T}}, \quad \bar{H}=\left[H^{\mathrm{T}}, 0,0,0,0,0\right]^{\mathrm{T}}, \quad \bar{M}=\left[M^{\mathrm{T}}, 0,0,0,0,0\right]^{\mathrm{T}},
\end{aligned}
$$

with

$$
\begin{aligned}
& \Gamma=\left(\begin{array}{cccccccc}
\Gamma_{11} & \Gamma_{12} & \Gamma_{13} & 0 & 0 & 0 & 0 & 0 \\
* & \Gamma_{22} & \Gamma_{23} & 0 & 0 & 0 & 0 & 0 \\
* & * & \Gamma_{33} & 0 & 0 & 0 & 0 & 0 \\
* & * & * & -R_{1} & 0 & 0 & 0 & 0 \\
* & * & * & * & -R_{3} & 0 & 0 & 0 \\
* & * & * & * & * & \Gamma_{66} & 0 & 0 \\
* & * & * & * & * & * & \Gamma_{77} & 0 \\
* & * & * & * & * & * & * & \Gamma_{88}
\end{array}\right), \\
& \Psi=(N(M-H)-N(D+I) 0 H-M-H D M D(N+H-M) D), \\
& \Gamma_{11}=P A_{0}+A_{0}^{\mathrm{T}} P+R_{1}+R_{2}+R_{3}+\tau_{2} R_{0}+\left(\epsilon_{1}+\epsilon_{2}\right) S_{0}^{\mathrm{T}} S_{0}+\epsilon_{3} T_{0}^{\mathrm{T}} T_{0}, \\
& \Gamma_{12}=P A_{1}-A_{0}^{\mathrm{T}} P D+\left(\epsilon_{1}+\epsilon_{2}\right) S_{0}^{\mathrm{T}} S_{1}+\epsilon_{3} T_{0}^{\mathrm{T}} T_{1}, \quad \Gamma_{13}=P A_{2}+\left(\epsilon_{1}+\epsilon_{2}\right) S_{0}^{\mathrm{T}} S_{2}+\epsilon_{3} T_{0}^{\mathrm{T}} T_{2}, \\
& \Gamma_{22}=R_{4}+R_{5}+R_{6}-(1-\bar{\tau}) R_{2}-D^{\mathrm{T}} P A_{1}-A_{1}^{\mathrm{T}} P D+\left(\epsilon_{1}+\epsilon_{2}\right) S_{1}^{\mathrm{T}} S_{1}+\epsilon_{3} T_{1}^{\mathrm{T}} T_{1}, \\
& \Gamma_{23}=-D^{\mathrm{T}} P A_{2}+\left(\epsilon_{1}+\epsilon_{2}\right) S_{1}^{\mathrm{T}} S_{2}+\epsilon_{3} T_{1}^{\mathrm{T}} T_{2}, \quad \Gamma_{33}=-\tau_{2} R_{0}+\left(\epsilon_{1}+\epsilon_{2}\right) S_{2}^{\mathrm{T}} S_{2}+\epsilon_{3} T_{2}^{\mathrm{T}} T_{2}, \\
& \Gamma_{66}=R_{4}, \quad \Gamma_{77}=R_{6}, \quad \Gamma_{88}=(1-\bar{\tau}) R_{5} .
\end{aligned}
$$

Proof. Choose a Lyapunov-Krasovskii functional candidate as

$$
V\left(t, \xi_{t}\right)=\sum_{i=0}^{4} V_{i}\left(t, \xi_{t}\right)
$$

where

$$
\begin{aligned}
& V_{0}\left(t, x_{t}\right)=\left[x(t)-D x\left(t_{\tau}\right)\right]^{\mathrm{T}} P\left[x(t)-D x\left(t_{\tau}\right)\right] \\
& V_{1}\left(t, x_{t}\right)=\int_{t_{\tau_{1}}}^{t}\left(\begin{array}{c}
x(s) \\
x(s-\tau(s))
\end{array}\right)^{\mathrm{T}}\left(\begin{array}{cc}
R_{1} & 0 \\
0 & R_{4}
\end{array}\right)\left(\begin{array}{c}
x(s) \\
x(s-\tau(s))
\end{array}\right) \mathrm{d} s
\end{aligned}
$$


Journal of Applied Mathematics

$$
\begin{gathered}
\quad+\int_{t_{\tau}}^{t}\left(\begin{array}{c}
x(s) \\
x(s-\tau(s))
\end{array}\right)^{\mathrm{T}}\left(\begin{array}{cc}
R_{2} & 0 \\
0 & R_{5}
\end{array}\right)\left(\begin{array}{c}
x(s) \\
x(s-\tau(s))
\end{array}\right) \mathrm{d} s \\
+\int_{t_{\tau_{2}}}^{t}\left(\begin{array}{c}
x(s) \\
x(s-\tau(s))
\end{array}\right)^{\mathrm{T}}\left(\begin{array}{cc}
R_{3} & 0 \\
0 & R_{6}
\end{array}\right)\left(\begin{array}{c}
x(s) \\
x(s-\tau(s))
\end{array}\right) \mathrm{d} s ; \\
V_{2}\left(t, x_{t}\right)=\int_{\tau_{\tau_{2}}}^{t}\left[s-\left(t-\tau_{2}\right)\right] x^{\mathrm{T}}(s) R_{0} x(s) \mathrm{d} s ; \\
V_{3}\left(t, x_{t}\right)=\tau_{2} \int_{-\tau_{2}}^{0} \int_{t+\theta}^{t} f^{\mathrm{T}}(s) R_{7} f(s) \mathrm{d} s \mathrm{~d} \theta+\delta \int_{-\tau_{2}}^{-\tau_{1}} \int_{t+\theta}^{t} f^{\mathrm{T}}(s) R_{8} f(s) \mathrm{d} s \mathrm{~d} \theta ; \\
V_{4}\left(t, x_{t}\right)=\int_{-\tau_{2}}^{0} \int_{t+\theta}^{t} \operatorname{tr}\left(g^{\mathrm{T}}(s) R_{9} g(s)\right) \mathrm{d} s \mathrm{~d} \theta+\int_{-\tau_{2}}^{-\tau_{1}} \int_{t+\theta}^{t} \operatorname{tr}\left(g^{\mathrm{T}}(s) R_{10} g(s)\right) \mathrm{d} s \mathrm{~d} \theta
\end{gathered}
$$

According to Itô's differential formula [1], the stochastic differential is

$$
\mathrm{d} V\left(t, x_{t}\right)=L V\left(t, x_{t}\right) \mathrm{d} t+2\left[x(t)-D x\left(t_{\tau}\right)\right]^{\mathrm{T}} P g(t) \mathrm{d} B(t),
$$

where

$$
L V\left(t, x_{t}\right) \mathrm{d} t=2\left[x(t)-D x\left(t_{\tau}\right)\right]^{\mathrm{T}} P f(t)+g(t)^{\mathrm{T}} P g(t)+\sum_{i=1}^{4} \dot{V}_{i}\left(t, x_{t}\right) .
$$

Direct computations give

$$
\begin{aligned}
\dot{V}_{1}\left(t, x_{t}\right) \leqslant & x^{\mathrm{T}}(t)\left(R_{1}+R_{2}+R_{3}\right) x(t)-x^{\mathrm{T}}\left(t_{\tau_{1}}\right) R_{1} x\left(t_{\tau_{1}}\right)-x^{\mathrm{T}}\left(t_{\tau_{2}}\right) R_{3} x\left(t_{\tau_{2}}\right) \\
& +x^{\mathrm{T}}\left(t_{\tau}\right)\left(R_{4}+R_{5}+R_{6}-(1-\bar{\tau}) R_{2}\right) x\left(t_{\tau}\right) \\
& -x^{\mathrm{T}}\left(t_{\tau_{1}}-\tau\left(t_{\tau_{1}}\right)\right) R_{4} x\left(t_{\tau_{1}}-\tau\left(t_{\tau_{1}}\right)\right) \\
& -(1-\bar{\tau}) x^{\mathrm{T}}\left(t_{\tau}-\tau\left(t_{\tau}\right)\right) R_{5} x\left(t_{\tau}-\tau\left(t_{\tau}\right)\right) \\
& -x^{\mathrm{T}}\left(t_{\tau_{2}}-\tau\left(t_{\tau_{2}}\right)\right) R_{6} x\left(t_{\tau_{2}}-\tau\left(t_{\tau_{2}}\right)\right) \quad\{b y \dot{\tau}(t) \leqslant \bar{\tau}\}
\end{aligned}
$$




$$
\begin{aligned}
\dot{V}_{2}\left(t, x_{t}\right) \leqslant & \tau_{2} x^{\mathrm{T}}(t) R_{0} x(t)-\int_{t_{\tau}}^{t} x^{\mathrm{T}}(s) R_{0} x(s) \mathrm{d} s \\
\leq & \tau_{2} x^{\mathrm{T}}(t) R_{0} x(t)-\left(\frac{1}{\tau_{2}} \int_{t_{\tau}}^{t} x(s) \mathrm{d} s\right)^{\mathrm{T}}\left(\tau_{2} R_{0}\right)\left(\frac{1}{\tau_{2}} \int_{t_{\tau}}^{t} x(s) \mathrm{d} s\right) ; \quad \text { by Lemma 2.5, } \\
\dot{V}_{3}\left(t, x_{t}\right)= & f^{\mathrm{T}}(t)^{\mathrm{T}}\left(\tau_{2}^{2} R_{7}+\delta^{2} R_{8}\right) f(t) \\
& -\tau_{2} \int_{t_{\tau_{2}}}^{t} f^{\mathrm{T}}(s)^{\mathrm{T}} R_{7} f(s) \mathrm{d} s-\delta \int_{t_{\tau_{2}}}^{t_{\tau_{1}}} f^{\mathrm{T}}(s)^{\mathrm{T}} R_{8} f(s) \mathrm{d} s ; \\
\dot{V}_{4}\left(t, x_{t}\right)= & \operatorname{tr}\left(g^{\mathrm{T}}(t)^{\mathrm{T}}\left(\tau_{2} R_{9}+\delta R_{10}\right) g(t)\right) \\
& -\int_{t_{\tau_{2}}}^{t} \operatorname{tr}\left(g^{\mathrm{T}}(s)^{\mathrm{T}} R_{9} g(s)\right) \mathrm{d} s-\int_{t_{\tau_{2}}}^{t_{\tau_{1}}} \operatorname{tr}\left(g^{\mathrm{T}}(s)^{\mathrm{T}} R_{10} g(s)\right) \mathrm{d} s .
\end{aligned}
$$

By (1) of Lemma 2.6, for any scalar $\epsilon_{1}>0$, we have

$$
\begin{aligned}
2\left[x(t)-D x\left(t_{\tau}\right)\right]^{\mathrm{T}} P f(t) & =2 \xi^{\mathrm{T}}(t) \bar{P} V_{1} \xi(t)+2 \xi^{\mathrm{T}}(t) \bar{P} G F(t) V_{3} \xi(t) \\
& \leq 2 \xi^{\mathrm{T}}(t) \bar{P} V_{1} \xi(t)+\epsilon_{1}^{-1} \xi^{\mathrm{T}}(t) \bar{P} G \star+\epsilon_{1} \xi^{\mathrm{T}}(t) V_{3}^{\mathrm{T}} \star
\end{aligned}
$$

and by (2) of Lemma 2.6, for any $\epsilon_{2}>0$ satisfying $\left[\tau_{2}^{2} R_{7}+\delta^{2} R_{8}\right]^{-1}-\epsilon_{2}^{-1} G G^{\mathrm{T}}>0$, we have

$$
\begin{aligned}
f^{\mathrm{T}}(t)\left[\tau_{2}^{2} R_{7}+\delta^{2} R_{8}\right] f(t) & =\xi^{\mathrm{T}}(t)\left(V_{1}+G F(t) V_{3}\right)^{\mathrm{T}} Z \star \\
& \leq \xi^{\mathrm{T}}(t) V_{1}^{\mathrm{T}}\left[Z^{-1}-\epsilon_{2}^{-1} G G^{T}\right]^{-1} \star+\epsilon_{2} \xi^{\mathrm{T}}(t) V_{3}^{\mathrm{T}} \star .
\end{aligned}
$$

For any scalar $\epsilon_{3}>0$ satisfying $\left[\tau_{2} R_{9}+\delta R_{10}\right]^{-1}-\epsilon_{3}^{-1} G G^{\mathrm{T}}>0$, the following inequality holds:

$$
\begin{aligned}
\operatorname{tr}\left(g^{\mathrm{T}}(t)\left[P+\tau_{2} R_{9}+\delta R_{10}\right] g(t)\right) & =g^{\mathrm{T}}(t)\left[P+\tau_{2} R_{9}+\delta R_{10}\right] g(t) \\
& =\xi^{\mathrm{T}}(t)\left(V_{2}+G F(t) V_{4}\right)^{\mathrm{T}} U \star \\
& \leq \xi^{\mathrm{T}}(t) V_{2}^{\mathrm{T}}\left[U^{-1}-\epsilon_{3}^{-1} G G^{\mathrm{T}}\right]^{-1} \star+\epsilon_{3} \xi^{\mathrm{T}}(t) V_{4}^{\mathrm{T}} \star .
\end{aligned}
$$


In addition, it follows from (3.4), (3) of Lemma 2.6 and Lemma 2.5 that

$$
\begin{aligned}
& -2 \xi^{\mathrm{T}}(t) N \int_{t_{\tau}}^{t} f(s) \mathrm{d} s \leqslant \xi^{\mathrm{T}}(t) N R_{7}^{-1} \star+\left(\int_{t_{\tau}}^{t} f(s) \mathrm{d} s\right)^{\mathrm{T}} R_{7} \star \\
& \leqslant \xi^{\mathrm{T}}(t) N R_{7}^{-1} \star+\tau_{2} \int_{t_{\tau}}^{t} f^{\mathrm{T}}(s) R_{7} f(s) \mathrm{d} s, \\
& -2 \xi^{\mathrm{T}}(t) H \int_{t_{\tau}}^{t_{\tau_{1}}} f(s) \mathrm{d} s \leqslant \xi^{\mathrm{T}}(t) H R_{8}^{-1} \star+\left(\int_{t_{\tau}}^{t_{\tau_{1}}} f(s) \mathrm{d} s\right)^{\mathrm{T}} R_{8} \star \\
& \leqslant \xi^{\mathrm{T}}(t) H R_{8}^{-1} \star+\delta \int_{t_{\tau}}^{t_{\tau_{1}}} f^{\mathrm{T}}(s) R_{8} f(s) \mathrm{d} s, \\
& -2 \xi^{\mathrm{T}}(t) M \int_{t_{\tau_{2}}}^{t_{\tau}} f(s) \mathrm{d} s \\
& \leqslant \xi^{\mathrm{T}}(t) M\left(R_{7}+R_{8}\right)^{-1} \star+\left(\int_{t_{\tau_{2}}}^{t_{\tau}} f(s) \mathrm{d} s\right)^{\mathrm{T}}\left(R_{7}+R_{8}\right) \star \\
& \leq \xi^{\mathrm{T}}(t) M\left(R_{7}+R_{8}\right)^{-1} \star+\int_{t_{\tau_{2}}}^{t_{\tau}} f^{\mathrm{T}}(s)\left(\tau_{2} R_{7}+\delta R_{8}\right) f(s) \mathrm{d} s, \\
& -2 \xi^{\mathrm{T}}(t) N \int_{t_{\tau}}^{t} g(s) \mathrm{d} B(s) \leq \xi^{\mathrm{T}}(t) N R_{9}^{-1} \star+\left(\int_{t_{\tau}}^{t} g(s) \mathrm{d} B(s)\right)^{\mathrm{T}} R_{9} \star, \\
& -2 \xi^{\mathrm{T}}(t) H \int_{t_{\tau}}^{t_{\tau_{1}}} g(s) \mathrm{d} B(s) \leq \xi^{\mathrm{T}}(t) H R_{10}^{-1} \star+\left(\int_{t_{\tau}}^{t_{\tau_{1}}} g(s) \mathrm{d} B(s)\right)^{\mathrm{T}} R_{10} \star, \\
& -2 \xi^{\mathrm{T}}(t) M \int_{t_{\tau_{2}}}^{t_{\tau}} g(s) \mathrm{d} B(s) \leq \xi^{\mathrm{T}}(t) M\left(R_{9}+R_{10}\right)^{-1} \star \\
& +\left(\int_{t_{\tau_{2}}}^{t_{\tau}} g(s) \mathrm{d} B(s)\right)^{\mathrm{T}}\left(R_{9}+R_{10}\right) \star
\end{aligned}
$$

Note that

$$
\begin{aligned}
& \mathbb{E}\left\{\left(\int_{t_{\tau}}^{t} g(s) \mathrm{d} B(s)\right)^{\mathrm{T}} R_{9} \star=\int_{t_{\tau}}^{t} \operatorname{tr}\left(g^{\mathrm{T}}(s) R_{9} g(s)\right) \mathrm{d} s,\right. \\
& \mathbb{E}\left\{\left(\int_{t_{\tau}}^{t_{\tau_{1}}} g(s) \mathrm{d} B(s)\right)^{\mathrm{T}} R_{10 \star}\right\}=\int_{t_{\tau}}^{t_{\tau_{1}}}\left(g^{\mathrm{T}}(s) R_{10} g(s)\right) \mathrm{d} s,
\end{aligned}
$$




$$
\mathbb{E}\left\{\left(\int_{t_{\tau_{2}}}^{t_{\tau}} g(s) \mathrm{d} B(s)\right)^{\mathrm{T}}\left(R_{9}+R_{10}\right) \star\right\}=\int_{t_{\tau_{2}}}^{t_{\tau}}\left(g^{\mathrm{T}}(s)\left(R_{9}+R_{10}\right) g(s)\right) \mathrm{d} s .
$$

Applying inequalities from (3.13) to (3.19) to (3.12), it yields

$$
\mathcal{L}_{\omega} V\left(t, \xi_{t}\right) \leqslant \xi^{\mathrm{T}}(t) \Lambda \xi(t)
$$

where

$$
\begin{aligned}
\Lambda= & \Theta+\epsilon_{1}^{-1} \bar{P} G(\bar{P} G)^{\mathrm{T}}+V_{1}^{\mathrm{T}}\left[Z^{-1}-\epsilon_{2}^{-1} G G^{\mathrm{T}}\right]^{-1} V_{1}+V_{2}^{\mathrm{T}}\left[U^{-1}-\epsilon_{3}^{-1} G G^{\mathrm{T}}\right]^{-1} V_{2} \\
& +N\left(R_{7}^{-1}+R_{9}^{-1}\right) N^{\mathrm{T}}+H\left(R_{8}^{-1}+R_{10}^{-1}\right) H^{\mathrm{T}}+M\left(\left(R_{7}+R_{8}\right)^{-1}+\left(R_{9}+R_{10}\right)^{-1}\right) M^{\mathrm{T}}
\end{aligned}
$$

with $\Theta$ being defined in Theorem 3.1.

By applying the Schur complement to (3.6) results in $\Lambda<0$, which implies

$$
L V\left(t, x_{t}\right) \leqslant \xi^{\mathrm{T}}(t) \Lambda \xi(t)<0
$$

therefore,

$$
L V\left(t, x_{t}\right) \leqslant-v \xi(t) \xi(t) \leqslant-v x^{\mathrm{T}}(t) x(t),
$$

where $v=\lambda_{m}\{-\Lambda\}$. Now, integrating both sides of (3.23) from 0 to $t>0$, and then taking the expectation, we obtain

$$
\mathbb{E}\left\{\left.V\left(s, x_{s}\right)\right|_{s=0} ^{s=t}\right\}=\mathbb{E}\left\{\int_{0}^{t} L V\left(s, x_{s}\right) \mathrm{d} s\right\} \leqslant-v \int_{0}^{t} \mathbb{E}\left\{x^{\mathrm{T}}(s) x(s)\right\} \mathrm{d} s .
$$

On the other hand, it follows from (3.9) that

$$
\mathbb{E}\left\{V\left(t, x_{t}\right)\right\} \geq \mathbb{E}\left\{\eta^{\mathrm{T}}(t) P \eta(t)\right\} \geq \lambda_{m}\{P\} \mathbb{E}\left\{\eta^{\mathrm{T}}(t) \eta(t)\right\}
$$

Therefore, by (3.24) and (3.25)

$$
\lambda_{m}\{P\} \mathbb{E}\left\{\eta^{\mathrm{T}}(t) \eta(t)\right\} \leqslant \mathbb{E}\left\{V\left(0, x_{0}\right)\right\}-v \int_{0}^{t} \mathbb{E}\left\{x^{\mathrm{T}}(s) x(s)\right\} \mathrm{d} s .
$$


Since $\rho(D)<1$, choose $\epsilon>0$ such that $e^{\tau_{2} \epsilon} \rho^{2}(D)<1$, which implies $\rho^{2}(D)<e^{-\tau_{2} \epsilon}<1$, denote $\epsilon_{0}=e^{-\tau_{2} \epsilon}$. Let $\sigma$ be any nonnegative real number. For all $0 \leqslant t \leqslant \sigma$, it follows from (1) of Lemma 2.6 that

$$
\begin{aligned}
\mathbb{E}\left\{\eta^{\mathrm{T}}(t) \eta(t)\right\} & \geq \mathbb{E} x^{\mathrm{T}}(t) x(t)-2 \mathbb{E}\left\{x^{\mathrm{T}}(t) D x\left(t_{\tau}\right)\right\}+\mathbb{E} x^{\mathrm{T}}\left(t_{\tau}\right) D^{\mathrm{T}} D x\left(t_{\tau}\right) \\
& \geq\left(1-\epsilon_{0}\right) \mathbb{E} x^{\mathrm{T}}(t) x(t)-\left(\epsilon_{0}^{-1}-1\right) \mathbb{E} x^{\mathrm{T}}\left(t_{\tau}\right) D^{\mathrm{T}} D x\left(t_{\tau}\right) .
\end{aligned}
$$

Hence

$$
\mathbb{E} x^{\mathrm{T}}(t) x(t) \leqslant \frac{1}{1-\epsilon_{0}} \mathbb{E}\left\{\eta^{\mathrm{T}}(t) \eta(t)\right\}+\frac{1}{\epsilon_{0}} \mathbb{E}\left\{x^{\mathrm{T}}\left(t_{\tau}\right) D^{\mathrm{T}} D x\left(t_{\tau}\right)\right\}
$$

By (3.25), (3.27), and (3.28), we then derive that for all $0 \leqslant t \leqslant \sigma$,

$$
\begin{aligned}
\mathbb{E} x^{\mathrm{T}}(t) x(t) & \leqslant \frac{1}{1-\epsilon_{0}} \sup _{0 \leqslant t \leqslant \sigma} \mathbb{E}\left\{\eta^{\mathrm{T}}(t) \eta(t)\right\}+\frac{1}{\epsilon_{0}} \sup _{0 \leqslant t \leqslant \sigma} \mathbb{E}\left\{x^{\mathrm{T}}\left(t_{\tau}\right) D^{\mathrm{T}} D x\left(t_{\tau}\right)\right\} \\
& \leqslant \frac{1}{\left(1-\epsilon_{0}\right)} \sup _{0 \leqslant t \leqslant \sigma} \mathbb{E}\left\{\eta^{\mathrm{T}}(t) \eta(t)\right\}+\frac{\lambda_{M}\left\{D^{\prime} D\right\}}{\epsilon_{0}} \sup _{-\tau_{2} \leqslant t \leqslant \sigma} \mathbb{E}\left\{x^{\mathrm{T}}(t) x(t)\right\} \\
& =\frac{1}{\left(1-\epsilon_{0}\right)} \sup _{0 \leqslant t \leqslant \sigma} \mathbb{E}\left\{\eta^{\mathrm{T}}(t) \eta(t)\right\}+\frac{\|D\|^{2}}{\epsilon_{0}} \sup _{-\tau_{2} \leqslant t \leqslant \sigma} \mathbb{E}\left\{x^{\mathrm{T}}(t) x(t)\right\} .
\end{aligned}
$$

However, this holds for all $-\tau \leqslant t \leqslant 0$ as well. Therefore,

$$
\sup _{-\tau_{2} \leqslant t \leqslant \sigma} \mathbb{E}\left\{x^{\mathrm{T}}(t) x(t)\right\} \leqslant \frac{1}{\left(1-\epsilon_{0}\right)} \sup _{0 \leqslant t \leqslant \sigma} \mathbb{E}\left\{\eta^{\mathrm{T}}(t) \eta(t)\right\}+\frac{\rho^{2}(D)}{\epsilon_{0}} \sup _{-\tau_{2} \leqslant t \leqslant \sigma} \mathbb{E}\left\{x^{\mathrm{T}}(t) x(t)\right\}
$$

Since $\rho^{2}(D)<\epsilon_{0}$ and $P>0$, we obtain that

$$
\frac{\left(1-\epsilon_{0}\right)\left(\epsilon_{0}-\rho^{2}(D)\right)}{\epsilon_{0}} \sup _{-\tau_{2} \leqslant t \leqslant \sigma} \mathbb{E}\left\{x^{\mathrm{T}}(t) x(t)\right\} \leqslant \sup _{0 \leqslant t \leqslant \sigma} \mathbb{E}\left\{\eta^{\mathrm{T}}(t) \eta(t)\right\} .
$$

By supremum property,

$$
\sup _{-\tau_{2} \leqslant t \leqslant \sigma} \mathbb{E}\left\{x^{\mathrm{T}}(t) x(t)\right\} \geq \sup _{0 \leqslant t \leqslant \sigma} \mathbb{E}\left\{x^{\mathrm{T}}(t) x(t)\right\}
$$

By (3.31) and (3.32),

$$
\frac{\left(1-\epsilon_{0}\right)\left(\epsilon_{0}-\rho^{2}(D)\right)}{\epsilon_{0}} \sup _{0 \leqslant t \leqslant \sigma} \mathbb{E}\left\{x^{\mathrm{T}}(t) x(t)\right\} \leqslant \sup _{0 \leqslant t \leqslant \sigma} \mathbb{E}\left\{\eta^{\mathrm{T}}(t) \eta(t)\right\} .
$$


For any $t \geq 0$, it follows from (3.26), (3.33) and $\sigma$ being an arbitrary nonnegative real number that

$$
\sup _{t \geq 0} \mathbb{E}\left\{x^{\mathrm{T}}(t) x(t)\right\} \leqslant \mu \mathbb{E}\left\{V\left(0, x_{0}\right)\right\}-v \mu \int_{0}^{t} \mathbb{E}\left\{x^{\mathrm{T}}(s) x(s)\right\} \mathrm{d} s .
$$

Since $\sigma$ is an arbitrary nonnegative number, we have

$$
\mathbb{E}\left\{x^{\mathrm{T}}(t) x(t)\right\} \leqslant \mu \mathbb{E}\left\{V\left(0, x_{0}\right)\right\}-v \mu \int_{0}^{t} \mathbb{E}\left\{x^{\mathrm{T}}(s) x(s)\right\} \mathrm{d} s, \quad \forall t \geq 0 .
$$

Then, applying Gronwall-Bellman lemma to (3.35), it yields

$$
\mathbb{E}\left\{x^{\mathrm{T}}(t) x(t)\right\} \leqslant \mu \mathbb{E}\left\{V\left(0, x_{0}\right)\right\} \mathrm{e}^{-v \mu t} .
$$

Note that there exists a scalar $\alpha>0$ such that

$$
\mu \mathbb{E}\left\{V\left(0, x_{0}\right)\right\} \leqslant \alpha \sup _{-\tau_{2} \leqslant \theta \leqslant 0} \mathbb{E}\{\phi(\theta)\}
$$

Denote $\mu=\epsilon_{0}\left[\left(1-\epsilon_{0}\right)\left(\epsilon_{0}-\rho^{2}(D)\right) \lambda_{m}\{P\}\right]^{-1}>0, \beta=v \mu>0$, then we have

$$
\mathbb{E}\left\{x^{\mathrm{T}}(t) x(t)\right\} \leqslant \alpha \mathrm{e}^{-\beta t} \sup _{-\tau_{2} \leqslant \theta \leqslant 0} \mathbb{E}\{\phi(\theta)\} .
$$

By Definition 2.4, system $(\Sigma)$ is robustly stochastically mean-square exponentially stable.

Remark 3.2. Theorem 3.1 provides a delay-dependent condition with a new LyapunovKrasovskii functional, which makes full use of the relationship among the time-varying delay, its upper and lower bounds, and their difference. It is noted that this condition is obtained by free-weighting matrices techniques; model transformation is not resorted to in the derivation of Theorem 3.1. Thus, the conservatism caused by model transformation will be reduced.

If $\tau_{1}=\tau_{2}=\tau_{0}$, then $\bar{\tau}=0, \tau(t)=\tau_{0}$. Applying Leibniz-Newton formula to (2.1), it yields the following zero equations:

$$
2 \zeta^{\mathrm{T}}(t) L\left[x(t)-D x\left(t-\tau_{0}\right)-x\left(t-\tau_{0}\right)+D x\left(t-2 \tau_{0}\right)-\int_{t_{\tau_{0}}}^{t} f(s) \mathrm{d} s-\int_{t_{\tau_{0}}}^{t} g(s) \mathrm{d} B(s)\right]=0
$$

where $L$ is any matrices with appropriate dimension, and

$$
\zeta^{\mathrm{T}}(t)=\left[x^{\mathrm{T}}(t), x^{\mathrm{T}}\left(t-\tau_{0}\right), \frac{1}{\tau_{0}} \int_{t-\tau_{0}}^{t} x^{\mathrm{T}}(s) \mathrm{d} s, x^{\mathrm{T}}\left(t-2 \tau_{0}\right)\right] .
$$

With zero equations (3.39) above, we obtain the following corollary. 
Corollary 3.3. For given constant delay $\tau_{0}$, system $(\Sigma)$ is robustly stochastically exponentially stable for all admissible uncertainties satisfying (2.4) and (2.5), if there exist symmetric positive definite matrices $P_{0}>0, Z_{0}>0, Z_{1}>0, Z_{2}>0, Z_{3}>0, Z_{4}>0$, scalars $\epsilon_{i}, i=1,2,3$ and any matrices $L$ with appropriate dimensions such that the following $L M I$ (3.41) holds:

$$
\Omega=\left(\begin{array}{cccccccc}
\Xi & \bar{P}_{0} G & \tau_{0}^{2} W_{1}^{\mathrm{T}} Z_{3} & 0 & \tau_{0} W_{2}^{\mathrm{T}} Z_{4} & 0 & \bar{L} & \bar{L} \\
* & -\epsilon_{1} I & 0 & 0 & 0 & 0 & 0 & 0 \\
* & * & -\tau_{0}^{2} Z_{3} & \tau_{0}^{2} Z_{3} G & 0 & 0 & 0 & 0 \\
* & * & * & -\epsilon_{2} I & 0 & 0 & 0 & 0 \\
* & * & * & * & -\tau_{0} Z_{4} & \tau_{0} Z_{4} G & 0 & 0 \\
* & * & * & * & * & -\epsilon_{3} I & 0 & 0 \\
* & * & * & * & * & * & -Z_{3} & 0 \\
* & * & * & * & * & * I & 0 & -Z_{4}
\end{array}\right)<0
$$

where

$$
\begin{gathered}
\Xi=\Pi+\Upsilon+\Upsilon^{\mathrm{T}}, \quad W_{1}=\left[\begin{array}{llll}
A_{0} & A_{1} & A_{2} & 0
\end{array}\right], \quad W_{2}=\left[\begin{array}{llll}
B_{0} & B_{1} & B_{2} & 0
\end{array}\right], \\
\bar{P}_{0}=\left[\begin{array}{llll}
P_{0} & -D P_{0} & 0 & 0
\end{array}\right]^{\mathrm{T}}, \quad \bar{L}=\left[\begin{array}{llll}
L^{\mathrm{T}} & 0 & 0 & 0
\end{array}\right]^{\mathrm{T}},
\end{gathered}
$$

with

$$
\begin{aligned}
& \Pi=\left(\begin{array}{cccc}
\Pi_{11} & \Pi_{12} & \Pi_{13} & 0 \\
* & \Pi_{22} & \Pi_{23} & 0 \\
* & * & \Pi_{33} & 0 \\
* & * & * & -Z_{2}
\end{array}\right), \quad \Upsilon=(L-L(D+I) \quad 0 \quad L D), \\
& \Pi_{11}=P_{0} A_{0}+A_{0}^{\mathrm{T}} P_{0}+Z_{1}+Z_{2}+\tau_{0} Z_{0}+\left(\epsilon_{1}+\epsilon_{2}\right) S_{0}^{\mathrm{T}} S_{0}+\epsilon_{3} T_{0}^{\mathrm{T}} T_{0}, \\
& \Pi_{12}=P_{0} A_{1}-A_{0}^{\mathrm{T}} D P_{0}+\left(\epsilon_{1}+\epsilon_{2}\right) S_{0}^{\mathrm{T}} S_{1}+\epsilon_{3} T_{0}^{\mathrm{T}} T_{1}, \\
& \Pi_{13}=P_{0} A_{2}+\left(\epsilon_{1}+\epsilon_{2}\right) S_{0}^{\mathrm{T}} S_{2}+\epsilon_{3} T_{0}^{\mathrm{T}} T_{2}, \\
& \Pi_{22}=-Z_{1}-P_{0} D^{\mathrm{T}} A_{1}-A_{1}^{\mathrm{T}} D P_{0}+\left(\epsilon_{1}+\epsilon_{2}\right) S_{1}^{\mathrm{T}} S_{1}+\epsilon_{3} T_{1}^{\mathrm{T}} T_{1}, \\
& \Pi_{23}=-P_{0} D^{\mathrm{T}} A_{2}+\left(\epsilon_{1}+\epsilon_{2}\right) S_{1}^{\mathrm{T}} S_{2}+\epsilon_{3} T_{1}^{\mathrm{T}} T_{2}, \quad \Pi_{33}=-\tau_{0} Z_{0}+\left(\epsilon_{1}+\epsilon_{2}\right) S_{2}^{\mathrm{T}} S_{2}+\epsilon_{3} T_{2}^{\mathrm{T}} T_{2} .
\end{aligned}
$$$$
\text { If } A_{2}=B_{2}=0, \Delta A_{2}(t)=\Delta B_{2}(t)=0, D=0 \text {, then, system (2.1) becomes }
$$$$
\mathrm{d} x(t)=\bar{f}(t) \mathrm{d} t+\bar{g}(t) \mathrm{d} B(t),
$$

where

$$
\begin{gathered}
\bar{f}(t)=\left(A_{0}+\Delta A_{0}(t)\right) x(t)+\left(A_{1}+\Delta A_{1}(t)\right) x\left(t_{\tau}\right), \\
\bar{g}(t)=\left(B_{0}+\Delta B_{0}(t)\right) x(t)+\left(B_{1}+\Delta B_{1}(t)\right) x\left(t_{\tau}\right) .
\end{gathered}
$$


Applying Leibniz-Newton formula to (3.44), it yields the following zero equations which will be used in our main result

$$
\begin{gathered}
2 \widetilde{\xi}^{\mathrm{T}}(t) \widehat{N}\left[x(t)-x\left(t_{\tau}\right)-\int_{t_{\tau}}^{t} \bar{f}(s) \mathrm{d} s-\int_{t_{\tau}}^{t} \bar{g}(s) \mathrm{d} B(s)\right]=0, \\
2 \widetilde{\xi}^{\mathrm{T}}(t) \widehat{H}\left[x\left(t_{\tau_{1}}\right)-x\left(t_{\tau}\right)-\int_{t_{\tau}}^{t_{\tau_{1}}} \bar{f}(s) \mathrm{d} s-\int_{t_{\tau}}^{t_{\tau_{1}}} \bar{g}(s) \mathrm{d} B(s)\right]=0, \\
2 \widetilde{\xi}^{\mathrm{T}}(t) \widehat{M}\left[x\left(t_{\tau}\right)-x\left(t_{\tau_{2}}\right)-\int_{t_{\tau_{2}}}^{t_{\tau}} \bar{f}(s) \mathrm{d} s-\int_{t_{\tau_{2}}}^{t_{\tau}} \bar{g}(s) \mathrm{d} B(s)\right]=0,
\end{gathered}
$$

where $N, H$, and $M$ are any matrices with appropriate dimensions, and

$$
\widetilde{\xi}^{\mathrm{T}}(t)=\left[x^{\mathrm{T}}(t), x^{\mathrm{T}}\left(t_{\tau}\right), x^{\mathrm{T}}\left(t_{\tau_{1}}\right), x^{\mathrm{T}}\left(t_{\tau_{2}}\right)\right]
$$

With zero equation (3.46) above, we obtain the following corollary.

Corollary 3.4. For given scalars $0 \leqslant \tau_{1} \leqslant \tau_{2}$ and $\bar{\tau}$, system (3.44) is robustly stochastically exponentially stable for all time-varying delays satisfying (2.3) and for all admissible uncertainties satisfying (2.4) and (2.5), if there exist symmetric positive definite matrices $P>0, R_{i}>0, i=$ $1,2, \ldots, 10$, scalars $\epsilon_{j}, j=1,2,3$ and any matrices $\widehat{N}, \widehat{H}, \widehat{M}$ with appropriate dimensions, such that the following LMI (3.48) holds:

$$
\widetilde{\Phi}=\left(\begin{array}{cc}
\widetilde{\Phi}_{1} & \widetilde{\Phi}_{2} \\
* & \widetilde{\Phi}_{3}
\end{array}\right)<0,
$$

where

$$
\begin{aligned}
& \widetilde{\Phi}_{1}=\left(\begin{array}{cccccc}
\Theta & \tilde{P} G & \tilde{V}_{1}^{T} Z & 0 & \tilde{V}_{2}^{T} U & 0 \\
* & -\epsilon_{1} I & 0 & 0 & 0 & 0 \\
* & * & -Z & Z G & 0 & 0 \\
* & * & * & -\epsilon_{2} I & 0 & 0 \\
* & * & * & * & -U & U G \\
* & * & * & * & * & -\epsilon_{3} I
\end{array}\right), \\
& \widetilde{\Phi}_{2}=\left(\begin{array}{llllll}
\widetilde{N} & \widetilde{H} & \widetilde{M} & \widetilde{N} & \widetilde{H} & \widetilde{M}
\end{array}\right),
\end{aligned}
$$

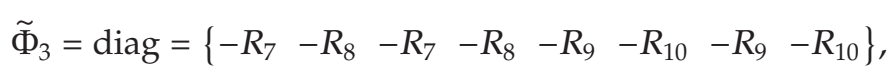

$$
\begin{aligned}
& \tilde{\Theta}=\tilde{\Gamma}+\tilde{\Psi}+\tilde{\Psi}^{\mathrm{T}}, \quad \tilde{V}_{1}=\left[\begin{array}{llll}
A_{0} & A_{1} & 0 & 0
\end{array}\right], \quad \tilde{V}_{2}=\left[\begin{array}{llll}
B_{0} & B_{1} & 0 & 0
\end{array}\right], \\
& \widetilde{P}=\left[\begin{array}{llll}
P & 0 & 0 & 0
\end{array}\right]^{\mathrm{T}}, \quad \mathrm{Z}=\tau_{2} R_{7}+\delta R_{8}, \quad U=P+\tau_{2} R_{9}+\delta R_{10}, \\
& \widetilde{N}=\left[\widehat{N}^{\mathrm{T}}, 0,0,0,0,0\right]^{\mathrm{T}}, \quad \widetilde{H}=\left[\widehat{H}^{\mathrm{T}}, 0,0,0,0,0\right]^{\mathrm{T}}, \quad \widetilde{M}=\left[\widehat{M}^{\mathrm{T}}, 0,0,0,0,0\right]^{\mathrm{T}} \text {, }
\end{aligned}
$$


with

$$
\begin{aligned}
& =\left(\begin{array}{cccc}
\widetilde{\Gamma}_{11} & \tilde{\Gamma}_{12} & 0 & 0 \\
* & \widetilde{\Gamma}_{22} & 0 & 0 \\
* & * & -R_{1} & 0 \\
* & * & * & -R_{3}
\end{array}\right), \\
\widetilde{\Psi} & =(\widehat{N} \widehat{M}-\widehat{H}-\widehat{N} \widehat{H}-\widehat{M}), \\
\widetilde{\Gamma}_{11} & =P A_{0}+A_{0}^{\mathrm{T}} P+R_{1}+R_{2}+R_{3}+\left(\epsilon_{1}+\epsilon_{2}\right) S_{0}^{\mathrm{T}} S_{0}+\epsilon_{3} T_{0}^{\mathrm{T}} T_{0}, \\
\tilde{\Gamma}_{12} & =P A_{1}+\left(\epsilon_{1}+\epsilon_{2}\right) S_{0}^{\mathrm{T}} S_{1}+\epsilon_{3} T_{0}^{\mathrm{T}} T_{1}, \quad \tilde{\Gamma}_{22}=-(1-\bar{\tau}) R_{2}+\left(\epsilon_{1}+\epsilon_{2}\right) S_{1}^{\mathrm{T}} S_{1}+\epsilon_{3} T_{1}^{\mathrm{T}} T_{1} .
\end{aligned}
$$

If $\tau_{1}=\tau_{2}=\tau_{0}, A_{2}=B_{2}=0, \Delta A_{i}(t)=\Delta B_{i}(t)=0, i=0,1,2$, then $\bar{\tau}=0, \tau(t)=\tau_{0}$ and (2.1) becomes

$$
\mathrm{d}\left[x(t)-D x\left(t-\tau_{0}\right)\right]=\widehat{f}(t) \mathrm{d} t+\widehat{g}(t) \mathrm{d} B(t),
$$

where

$$
\widehat{f}(t)=A_{0} x(t)+A_{1} x\left(t-\tau_{0}\right), \quad \widehat{g}(t)=B_{0} x(t)+B_{1} x\left(t-\tau_{0}\right) .
$$

Applying Leibniz-Newton formula to (3.51), it yields the following zero equations

$$
2 \varsigma^{\mathrm{T}}(t) \widehat{L}\left[x(t)-D x\left(t-\tau_{0}\right)-x\left(t-\tau_{0}\right)+D x\left(t-2 \tau_{0}\right)-\int_{t_{\tau_{0}}}^{t} \widehat{f}(s) \mathrm{d} s-\int_{t_{\tau_{0}}}^{t} \widehat{g}(s) \mathrm{d} B(s)\right]=0,
$$

where $\widehat{L}$ is any matrices with appropriate dimension, and

$$
\varsigma^{\mathrm{T}}(t)=\left[x^{\mathrm{T}}(t), x^{\mathrm{T}}\left(t-\tau_{0}\right), x^{\mathrm{T}}\left(t-2 \tau_{0}\right)\right]
$$

With zero equations (3.53) above, we obtain the following corollary.

Corollary 3.5. For given constant delay $\tau_{0}$, system (3.51) is robustly stochastically exponentially stable, if there exist symmetric positive definite matrices $P_{0}>0, Z_{1}>0, Z_{2}>0, Z_{3}>0, Z_{4}>0$, any matrices $\widehat{L}$ with appropriate dimensions, such that the following $L M I$ (3.41) holds:

$$
\widetilde{\Omega}=\left(\begin{array}{ccccc}
\widetilde{\Xi} & \tau_{0}^{2} \widetilde{W}_{1}^{\mathrm{T}} Z_{3} & \tau_{0} \widetilde{W}_{2}^{\mathrm{T}} Z_{4} & \widetilde{L} & \widetilde{L} \\
* & -\tau_{0}^{2} Z_{3} & 0 & 0 & 0 \\
* & * & -\tau_{0} Z_{4} & 0 & 0 \\
* & * & * & -Z_{3} & 0 \\
* & * & * & * & -Z_{4}
\end{array}\right)<0
$$


where

$$
\widetilde{\Xi}=\tilde{\Pi}+\tilde{\Upsilon}+\tilde{\Upsilon}^{\mathrm{T}}, \quad \widetilde{W}_{1}=\left[\begin{array}{lll}
A_{0} & A_{1} & 0
\end{array}\right], \quad \widetilde{W}_{2}=\left[\begin{array}{lll}
B_{0} & B_{1} & 0
\end{array}\right], \quad \widetilde{L}=\left[\begin{array}{lll}
\widehat{L}^{\mathrm{T}} & 0 & 0
\end{array}\right]^{\mathrm{T}},
$$

with

$$
\begin{aligned}
& \tilde{\Pi}=\left(\begin{array}{ccc}
\tilde{\Pi}_{11} & \tilde{\Pi}_{12} & 0 \\
* & \tilde{\Pi}_{22} & 0 \\
* & * & -Z_{2}
\end{array}\right), \quad \tilde{\Upsilon}=(\widehat{L}-\widehat{L}(D+I) \widehat{L} D), \\
& \tilde{\Pi}_{11}=P_{0} A_{0}+A_{0}^{\mathrm{T}} P_{0}+Z_{1}+Z_{2}, \quad \tilde{\Pi}_{12}=P_{0} A_{1}-A_{0}^{\mathrm{T}} D P_{0}, \\
& \tilde{\Pi}_{22}=-Z_{1}-P_{0} D^{\mathrm{T}} A_{1}-A_{1}^{\mathrm{T}} D P_{0} .
\end{aligned}
$$

Deterministic systems may be regarded as special class of stochastic systems, let $B_{i}=$ $0, \Delta B_{i}=0, i=0,1,2$, then system (2.1)-(2.2) becomes the following uncertain neutral system with both discrete and distributed interval time-varying delays

$$
\begin{gathered}
\left(\Sigma_{0}\right): \dot{x}(t)-D \dot{x}\left(t_{\tau}\right)=\left[A_{0}+\Delta A_{0}(t)\right] x(t)+\left[A_{1}+\Delta A_{1}(t)\right] x\left(t_{\tau}\right)+\left[A_{2}+\Delta A_{2}(t)\right] \int_{t_{\tau}}^{t} x(s) \mathrm{d} s, \\
x(t)=\phi(t), \quad t \in\left[-\tau_{2}, 0\right]
\end{gathered}
$$

Applying Leibniz-Newton formula to (3.58), it yields the following zero equations which will be used in our main result:

$$
\begin{gathered}
2 \xi^{\mathrm{T}}(t) N\left[x(t)-D x\left(t_{\tau}\right)-x\left(t_{\tau}\right)+D x\left(t_{\tau}-\tau\left(t_{\tau}\right)\right)-\int_{t_{\tau}}^{t} f(s) \mathrm{d} s\right]=0, \\
2 \xi^{\mathrm{T}}(t) H\left[x\left(t_{\tau_{1}}\right)-D x\left(t_{\tau_{1}}-\tau\left(t_{\tau_{1}}\right)\right)-x\left(t_{\tau}\right)+D x\left(t_{\tau}-\tau\left(t_{\tau}\right)\right)-\int_{t_{\tau}}^{t_{\tau_{1}}} f(s) \mathrm{d} s\right]=0, \\
2 \xi^{\mathrm{T}}(t) M\left[x\left(t_{\tau}\right)-D x\left(t_{\tau}-\tau\left(t_{\tau}\right)\right)-x\left(t_{\tau_{2}}\right)+D x\left(t_{\tau_{2}}-\tau\left(t_{\tau_{2}}\right)\right)-\int_{t_{\tau_{2}}}^{t_{\tau}} f(s) \mathrm{d} s\right]=0,
\end{gathered}
$$

where $N, H$, and $M$ are any matrices with appropriate dimensions, and $\xi(t)$ is defined in (3.5). With zero equations (3.60) above, we obtain

Corollary 3.6. For given scalars $0 \leqslant \tau_{1} \leqslant \tau_{2}$ and $\bar{\tau}$, system $\left(\Sigma_{0}\right)$ is robustly stochastically exponentially stable for all time-varying delays satisfying (2.3) and for all admissible uncertainties satisfying (2.4) and (2.5), if there exist symmetric positive definite matrices $P>0, R_{i}>0$, 
Journal of Applied Mathematics

$i=0,1,2, \ldots, 8$, scalars $\epsilon_{j}, j=1,2,3$ and any matrices $N, H, M$ with appropriate dimensions, such that the following LMI (3.61) holds:

$$
\Phi=\left(\begin{array}{ccccccc}
\Theta & \bar{P} G & V_{1}^{\mathrm{T}} Z & 0 & N & H & M \\
* & -\epsilon_{1} I & 0 & 0 & 0 & 0 & 0 \\
* & * & -Z & Z G & 0 & 0 & 0 \\
* & * & * & -\epsilon_{2} I & 0 & 0 & 0 \\
* & * & * & * & -R_{7} & 0 & 0 \\
* & * & * & * & * & -R_{8} & 0 \\
* & * & * & * & * & * & -R_{7}-R_{8}
\end{array}\right)<0
$$

where

$$
\begin{aligned}
& \Theta=\Gamma+\Psi+\Psi^{\mathrm{T}}, \quad V_{1}=\left[\begin{array}{llllllll}
A_{0} & A_{1} & A_{2} & 0 & 0 & 0 & 0 & 0
\end{array}\right], \quad V_{2}=\left[\begin{array}{llllllll}
B_{0} & B_{1} & B_{2} & 0 & 0 & 0 & 0 & 0
\end{array}\right], \\
& \bar{P}=\left[\begin{array}{llllllll}
P & -P D & 0 & 0 & 0 & 0 & 0 & 0
\end{array}\right]^{\mathrm{T}}, \quad Z=\tau_{2} R_{7}+\delta R_{8}, \quad U=P+\tau_{2} R_{9}+\delta R_{10},
\end{aligned}
$$

with

$$
\begin{aligned}
& \Gamma=\left(\begin{array}{cccccccc}
\Gamma_{11} & \Gamma_{12} & \Gamma_{13} & 0 & 0 & 0 & 0 & 0 \\
* & \Gamma_{22} & \Gamma_{23} & 0 & 0 & 0 & 0 & 0 \\
* & * & \Gamma_{33} & 0 & 0 & 0 & 0 & 0 \\
* & * & * & -R_{1} & 0 & 0 & 0 & 0 \\
* & * & * & * & -R_{3} & 0 & 0 & 0 \\
* & * & * & * & * & \Gamma_{66} & 0 & 0 \\
* & * & * & * & * & * & \Gamma_{77} & 0 \\
* & * & * & * & * & * & * & \Gamma_{88}
\end{array}\right), \\
& \Psi=(N(M-H)-N(D+I) 0 H-M-H D M D(N+H-M) D), \\
& \Gamma_{11}=P A_{0}+A_{0}^{\mathrm{T}} P+R_{1}+R_{2}+R_{3}+\tau_{2} R_{0}+\left(\epsilon_{1}+\epsilon_{2}\right) S_{0}^{\mathrm{T}} S_{0}+\epsilon_{3} T_{0}^{\mathrm{T}} T_{0}, \\
& \Gamma_{12}=P A_{1}-A_{0}^{\mathrm{T}} P D+\left(\epsilon_{1}+\epsilon_{2}\right) S_{0}^{\mathrm{T}} S_{1}+\epsilon_{3} T_{0}^{\mathrm{T}} T_{1}, \quad \Gamma_{13}=P A_{2}+\left(\epsilon_{1}+\epsilon_{2}\right) S_{0}^{\mathrm{T}} S_{2}+\epsilon_{3} T_{0}^{\mathrm{T}} T_{2}, \\
& \Gamma_{22}=R_{4}+R_{5}+R_{6}-(1-\bar{\tau}) R_{2}-D^{\mathrm{T}} P A_{1}-A_{1}^{\mathrm{T}} P D+\left(\epsilon_{1}+\epsilon_{2}\right) S_{1}^{\mathrm{T}} S_{1}+\epsilon_{3} T_{1}^{\mathrm{T}} T_{1}, \\
& \Gamma_{23}=-D^{\mathrm{T}} P A_{2}+\left(\epsilon_{1}+\epsilon_{2}\right) S_{1}^{\mathrm{T}} S_{2}+\epsilon_{3} T_{1}^{\mathrm{T}} T_{2}, \quad \Gamma_{33}=-\tau_{2} R_{0}+\left(\epsilon_{1}+\epsilon_{2}\right) S_{2}^{\mathrm{T}} S_{2}+\epsilon_{3} T_{2}^{\mathrm{T}} T_{2}, \\
& \Gamma_{66}=-R_{4}, \quad \Gamma_{77}=-R_{6}, \quad \Gamma_{88}=-(1-\bar{\tau}) R_{5} .
\end{aligned}
$$

Proof. Choose a Lyapunov-Krasovskii functional candidate as

$$
V\left(t, \xi_{t}\right)=\sum_{i=0}^{3} V_{i}\left(t, \xi_{t}\right)
$$

where $V_{i}\left(t, \xi_{t}\right), i=0,1,2,3$ are defined in (3.9). It can be concluded from Theorem 3.1. 
If $\tau_{1}=\tau_{2}=\tau_{0}, A_{2}=0, B_{i}=\Delta A_{i}(t)=\Delta B_{i}(t)=0, i=0,1,2$, then $\bar{\tau}=0, \tau(t)=\tau_{0}$ and (2.1) becomes

$$
\dot{x}(t)-D \dot{x}\left(t-\tau_{0}\right)=A_{0} x(t)+A_{1} x\left(t-\tau_{0}\right) .
$$

Applying Leibniz-Newton formula to (3.65), it yields the following zero equations:

$$
2 \varsigma^{\mathrm{T}}(t) \widehat{L}\left[x(t)-D x\left(t-\tau_{0}\right)-x\left(t-\tau_{0}\right)+D x\left(t-2 \tau_{0}\right)-\int_{t_{\tau_{0}}}^{t} \widehat{f}(s) \mathrm{d} s\right]=0,
$$

where $\widehat{L}$ is any matrices with appropriate dimension, $\widehat{f}(t)=A_{0} x(t)+A_{1} x\left(t-\tau_{0}\right)$, and

$$
S^{\mathrm{T}}(t)=\left[x^{\mathrm{T}}(t), x^{\mathrm{T}}\left(t-\tau_{0}\right), x^{\mathrm{T}}\left(t-2 \tau_{0}\right)\right]
$$

With zero equations (3.66) above, we obtain the following corollary.

Corollary 3.7. For given constant delay $\tau_{0}$, system (3.65) is robustly stochastically exponentially stable, if there exist symmetric positive definite matrices $P_{0}>0, Z_{1}>0, Z_{2}>0, Z_{3}>0, Z_{4}>0$, and any matrices $\widehat{L}$ with appropriate dimensions, such that the following $L M I$ (3.68) holds:

$$
\widetilde{\Omega}=\left(\begin{array}{cccc}
\widetilde{\Xi} & \tau_{0}^{2} \widetilde{W}_{1}^{\mathrm{T}} Z_{3} & \breve{L} & \breve{L} \\
* & -\tau_{0}^{2} Z_{3} & 0 & 0 \\
* & * & -Z_{3} & 0 \\
* & * & * & -Z_{4}
\end{array}\right)<0,
$$

where

$$
\widetilde{\Xi}=\tilde{\Pi}+\tilde{\Upsilon}+\tilde{\Upsilon}^{\mathrm{T}}, \quad \widetilde{W}_{1}=\left[\begin{array}{lll}
A_{0} & A_{1} & 0
\end{array}\right], \quad \check{L}=\left[\begin{array}{lll}
\widehat{L}^{\mathrm{T}} & 0 & 0
\end{array}\right]^{\mathrm{T}},
$$

with

$$
\begin{aligned}
& \tilde{\Pi}=\left(\begin{array}{ccc}
\widetilde{\Pi}_{11} & \tilde{\Pi}_{12} & 0 \\
* & \tilde{\Pi}_{22} & 0 \\
* & * & -Z_{2}
\end{array}\right), \quad \tilde{\Upsilon}=(\widehat{L}-\widehat{L}(D+I) \widehat{L} D), \\
& \tilde{\Pi}_{11}=P_{0} A_{0}+A_{0}^{\mathrm{T}} P_{0}+Z_{1}+Z_{2}, \quad \tilde{\Pi}_{12}=P_{0} A_{1}-A_{0}^{\mathrm{T}} D P_{0}, \\
& \tilde{\Pi}_{22}=-Z_{1}-P_{0} D^{\mathrm{T}} A_{1}-A_{1}^{\mathrm{T}} D P_{0} .
\end{aligned}
$$




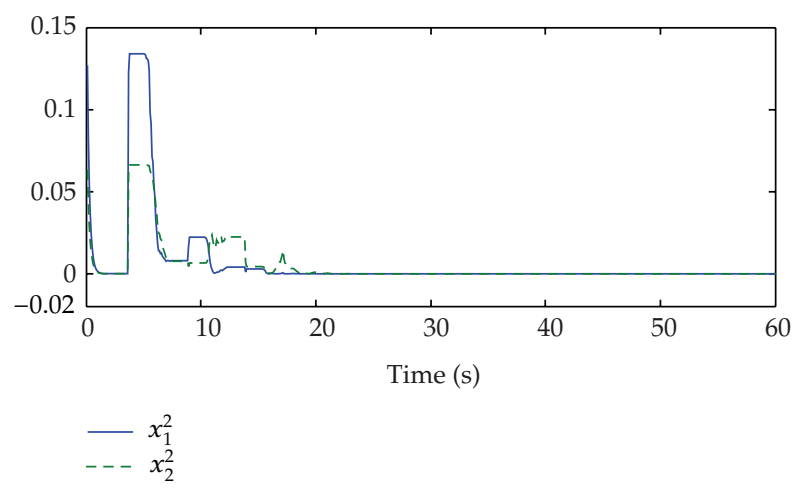

Figure 1: Mean-square exponential stability of the neutral stochastic system with interval time-varying delay.

Table 1: Allowable lower bounds and upper bounds for different $\bar{\tau}$.

\begin{tabular}{lcccccc}
\hline $\bar{\tau}$ & 0.3 & 0.6 & 1.0 & 1.2 & 1.5 & 1.52 \\
\hline$\tau_{2}$ & 1.5970 & 1.6724 & 1.8719 & 1.9717 & 2.1215 & 2.1315 \\
$\tau_{1}$ & 0.1500 & 0.3000 & 0.5000 & 0.6000 & 0.7500 & 0.7600 \\
$\delta$ & 1.4470 & 1.3724 & 1.3719 & 1.3717 & 1.3715 & 1.3715 \\
\hline
\end{tabular}

\section{Numerical Examples}

Example 4.1. Consider the uncertain neutral linear stochastic time-varying delay system $(\Sigma)$ with

$$
\begin{aligned}
& A_{0}=\left[\begin{array}{cc}
-2.0 & 0.0 \\
0.0 & -1.9
\end{array}\right], \quad A_{1}=\left[\begin{array}{cc}
-1.0 & 0.0 \\
-1.0 & -1.0
\end{array}\right], \quad A_{2}=-I, \quad D=-0.5 I, \\
& B_{0}=-0.1 I, \quad B_{1}=0.2 I, \quad B_{2}=0.1 I, \quad G=0.1 I, \quad S_{i}=T_{i}=0.1 I, \quad i=0,1,2,
\end{aligned}
$$

and $\tau(t)=\alpha \exp \left(-1 /(1+t)^{2}\right), 0 \leqslant \alpha \leqslant 2.1315$, set $\alpha=1$, in this situation, $\tau_{1}=0.3679, \tau_{2}=$ $1, \bar{\tau}=0.7358, \delta=0.6321$. Set $\phi(\theta)=\left[x_{1}^{\mathrm{T}}(\theta), x_{2}^{\mathrm{T}}(\theta)\right]^{\mathrm{T}}$, where $x_{1}(\theta), x_{2}(\theta)$ are random initial values with zero mean and variance 1.0, and $F(t)=\sin (t)$, the trajectories of $x_{1}(t)$ and $x_{2}(t)$ are shown in Figure 1. According to Theorem 3.1, the lower bounds and the upper bounds on the time delay to guarantee the system is robustly stochastically mean-square exponentially stable are listed in Table 1.

Example 4.2. Consider the uncertain linear stochastic system (3.44) with

$$
\begin{aligned}
& A_{0}=\left[\begin{array}{cc}
-2.0 & 0.0 \\
0.0 & -0.9
\end{array}\right], \quad A_{1}=\left[\begin{array}{cc}
-1.0 & 0.0 \\
-1.0 & -1.0
\end{array}\right], \\
& B_{0}=B_{1}=0, \quad G=0.2 I, \quad S_{i}=T_{i}=I, \quad i=0,1,2 .
\end{aligned}
$$


Table 2: Allowable upper bounds of $\tau_{2}$ for different $\bar{\tau}$.

\begin{tabular}{lccr}
\hline $\bar{\tau}$ & 0.3 & 0.5 & 0.9 \\
\hline Yan et al. [15] & 0.7288 & 0.5252 & 0.1489 \\
Corollary 3.4 & 0.9012 & 0.7498 & 0.5640 \\
\hline
\end{tabular}

Table 3: Allowable upper bounds of $\tau_{2}$ for different $c$.

\begin{tabular}{lcccccc}
\hline$c$ & 0 & 0.1 & 0.3 & 0.5 & 0.7 & 0.9 \\
\hline Fridman and Shaked [19] & 4.47 & 3.49 & 2.06 & 1.14 & 0.54 & 0.13 \\
Corollary 3.7 & 4.47 & 4.05 & 3.29 & 2.58 & 1.85 & 0.92 \\
\hline
\end{tabular}

According to Corollary 3.4, for $\tau_{1}=0$, the upper bounds on the time delay to guarantee the system is robustly stochastically mean-square exponentially stable are listed in Table 2 . At the same time, Table 2 also lists the upper bounds obtained from the criterion in [15].

Example 4.3. Consider the uncertain neutral linear stochastic system (3.51) with

$$
\begin{array}{ll}
A_{0}=\left[\begin{array}{ll}
0.5 & 0.0 \\
0.0 & 0.3
\end{array}\right], & A_{1}=\left[\begin{array}{cc}
-1.0 & 0.0 \\
-1.0 & -1.0
\end{array}\right], \\
D=\left[\begin{array}{cc}
-0.2 & 0.0 \\
-0.95 & 0.2
\end{array}\right], \quad B_{0}=0.2 I, \quad B_{1}=0.3 I .
\end{array}
$$

If Corollary 3.5 in this paper is applied, the maximal admissible delay $\tau_{M}$ of this example is $\tau_{M}=0.6001$.

Example 4.4. Consider the uncertain neutral linear stochastic system (3.65) with

$$
A_{0}=\left[\begin{array}{cc}
-2.0 & 0.0 \\
0.0 & -0.9
\end{array}\right], \quad A_{1}=\left[\begin{array}{cc}
-1.0 & 0.0 \\
-1.0 & -1.0
\end{array}\right], \quad D=\left[\begin{array}{ll}
c & 0 \\
0 & c
\end{array}\right], \quad 0 \leqslant c<1
$$

The maximum upper bound $\tau_{M}$ for this system in case of different $c$ s is listed in Table 3, which shows that the results obtained by the methods proposed in this paper are less conservative than that in [19].

\section{Conclusion}

The mean-square exponential stability for uncertain neutral stochastic system with both discrete and distributed time-varying delays has been investigated in this paper. Sufficient conditions have been established without ignoring any terms in the weak infinitesimal operator of Lyapunov-Krasovskii functional by considering the relationship among the timevarying delay, its upper bounds, and their difference. The usual restriction that $\dot{\tau}(t)<1$ has been removed by free-weight matrices method. According to the numerical examples, it has been shown that the proposed results improve some existing ones. 


\section{Acknowledgments}

The authors would like to thank the associate editor and the reviewers for their valuable and constructive comments and suggestions to improve the quality of this paper. This work is supported by National Natural Science Foundation of China (Grant nos. 60874114, 60904032, 61273126, 11201495), Natural Science Foundation of Guangdong Province (Grant no. 10251064101000008), Doctoral fund of Ministry of Education of China (Grant no. 20090171120022), and Principal Fund of South China Agricultural University (Grant no. 2009X008).

\section{References}

[1] X. R. Mao, Stochastic Differential Equations with Applications, Horwood, 2nd edition, 2008.

[2] L. R. Huang and X. R. Mao, "Delay-dependent exponential stability of neutral stochastic delay systems," IEEE Transactions on Automatic Control, vol. 54, no. 1, pp. 147-152, 2009.

[3] W.-H. Chen, W. X. Zheng, and Y. Shen, "Delay-dependent stochastic stability and $H_{\infty}$-control of uncertain neutral stochastic systems with time delay," IEEE Transactions on Automatic Control, vol. 54, no. 7, pp. 1660-1667, 2009.

[4] W. Feng and H. X. Wu, "Global asymptotic stability analysis for stochastic neutral-type delayed neural networks," in Proceedings of the Chinese Control and Decision Conference (CCDC '10), pp. 2658-2662, Xuzhou, China, May 2010.

[5] X. D. Li, "Global robust stability for stochastic interval neural networks with continuously distributed delays of neutral type," Applied Mathematics and Computation, vol. 215, no. 12, pp. 4370-4384, 2010.

[6] Y. Chen, W. X. Zheng, and A. K. Xue, "A new result on stability analysis for stochastic neutral systems," Automatica, vol. 46, no. 12, pp. 2100-2104, 2010.

[7] B. Song, S. Xu, J. Xia, Y. Zou, and Y. Chu, "Design of robust non-fragile $H_{\infty}$ filters for uncertain neutral stochastic systems with distributed delays," Asian Journal of Control, vol. 12, no. 1, pp. 39-45, 2010.

[8] G. Chen and Y. Shen, "Robust $H_{\infty}$ filter design for neutral stochastic uncertain systems with timevarying delay," Journal of Mathematical Analysis and Applications, vol. 353, no. 1, pp. 196-204, 2009.

[9] W.-H. Chen, Z.-H. Guan, and X. Lu, "Delay-dependent exponential stability of uncertain stochastic systems with multiple delays: an LMI approach," Systems \& Control Letters, vol. 54, no. 6, pp. 547-555, 2005.

[10] Y. He, Q.-G. Wang, L. Xie, and C. Lin, “Further improvement of free-weighting matrices technique for systems with time-varying delay," IEEE Transactions on Automatic Control, vol. 52, no. 2, pp. 293-299, 2007.

[11] Y. He, Q.-G. Wang, C. Lin, and M. Wu, “Delay-range-dependent stability for systems with time-varying delay," Automatica, vol. 43, no. 2, pp. 371-376, 2007.

[12] Y. Zhang, Y. He, and M. Wu, "Delay-dependent robust stability for uncertain stochastic systems with interval time-varying delay," Acta Automatica Sinica, vol. 35, no. 5, pp. 577-582, 2009.

[13] D. Yue, Q.-L. Han, and J. Lam, "Network-based robust $H_{\infty}$ control of systems with uncertainty," Automatica, vol. 41, no. 6, pp. 999-1007, 2005.

[14] X. F. Jiang and Q.-L. Han, "Delay-dependent robust stability for uncertain linear systems with interval time-varying delay," Automatica, vol. 42, no. 6, pp. 1059-1065, 2006.

[15] H. C. Yan, X. H. Huang, H. Zhang, and M. Wang, "Delay-dependent robust stability criteria of uncertain stochastic systems with time-varying delay," Chaos, Solitons and Fractals, vol. 40, no. 4, pp. 1668$1679,2009$.

[16] H. Y. Shao, "Improved delay-dependent stability criteria for systems with a delay varying in a range," Automatica, vol. 44, no. 12, pp. 3215-3218, 2008.

[17] J. J. Yu, K. J. Zhang, and S. M. Fei, "Further results on mean square exponential stability of uncertain stochastic delayed neural networks," Communications in Nonlinear Science and Numerical Simulation, vol. 14, no. 4, pp. 1582-1589, 2009.

[18] V. Suplin, E. Fridman, and U. Shaked, " $H_{\infty}$ control of linear uncertain time-delay systems-a projection approach," IEEE Transactions on Automatic Control, vol. 51, no. 4, pp. 680-685, 2006.

[19] E. Fridman and U. Shaked, "Delay-dependent stability and $H_{\infty}$ control: constant and time-varying delays," International Journal of Control, vol. 76, no. 1, pp. 48-60, 2003. 
[20] A. E. Rodkina and M. V. Basin, “On delay-dependent stability for vector nonlinear stochastic delaydifference equations with Volterra diffusion term," Systems \& Control Letters, vol. 56, no. 6, pp. 423430, 2007.

[21] M. V. Basin and A. E. Rodkina, "On delay-dependent stability for a class of nonlinear stochastic systems with multiple state delays," Nonlinear Analysis, vol. 68, no. 8, pp. 2147-2157, 2008.

[22] H. J. Gao and C. H. Wang, "A delay-dependent approach to robust $H_{\infty}$ filtering for uncertain discretetime state-delayed systems," IEEE Transactions on Signal Processing, vol. 52, no. 6, pp. 1631-1640, 2004.

[23] T. Li, A. G. Song, and S. M. Fei, "Robust stability of stochastic Cohen-Grossberg neural networks with mixed time-varying delays," Neurocomputing, vol. 73, no. 1-3, pp. 542-551, 2009.

[24] C. X. Li, J. T. Sun, and R. Y. Sun, "Stability analysis of a class of stochastic differential delay equations with nonlinear impulsive effects," Journal of the Franklin Institute, vol. 347, no. 7, pp. 1186-1198, 2010.

[25] C. X. Li, J. P. Shi, and J. T. Sun, "Stability of impulsive stochastic differential delay systems and its application to impulsive stochastic neural networks," Nonlinear Analysis, vol. 74, no. 10, pp. 3099 3111, 2011.

[26] K. Q. Gu, Q.-L. Han, A. C. J. Luo, and S.-I. Niculescu, “Discretized Lyapunov functional for systems with distributed delay and piecewise constant coefficients," International Journal of Control, vol. 74, no. 7, pp. 737-744, 2001.

[27] J. K. Hale and S. Lunel, Introduction to Functional Differential Equations, Springer, New York, NY, USA, 1993.

[28] E. K. Boukas and Z. Liu, Deterministic and Stochastic Time-Delay Systems, Birkhäuser, Boston, Mass, USA, 2002.

[29] V. Kolmanovskii and A. Myshkis, Introduction to the Theory and Applications of Functional Differential Equations, Kluwer Academic, Dodrecht, The Netherlands, 1999.

[30] K. Gu, "An integral inequality in the stability problem of time-delay systems," in Proceedings of the 39th IEEE Confernce on Decision and Control, vol. 3, pp. 2805-2810, Sydney, Australia, December 2000.

[31] S. Y. Xu and T. W. Chen, "Robust $H_{\infty}$ control for uncertain stochastic systems with state delay," IEEE Transactions on Automatic Control, vol. 47, no. 12, pp. 2089-2094, 2002. 


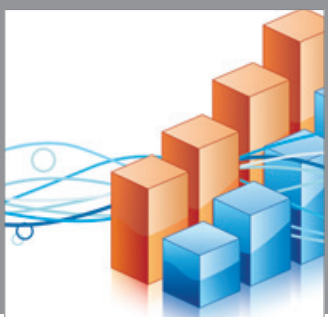

Advances in

Operations Research

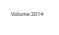

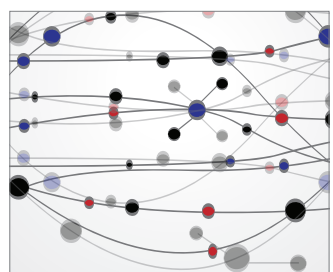

\section{The Scientific} World Journal
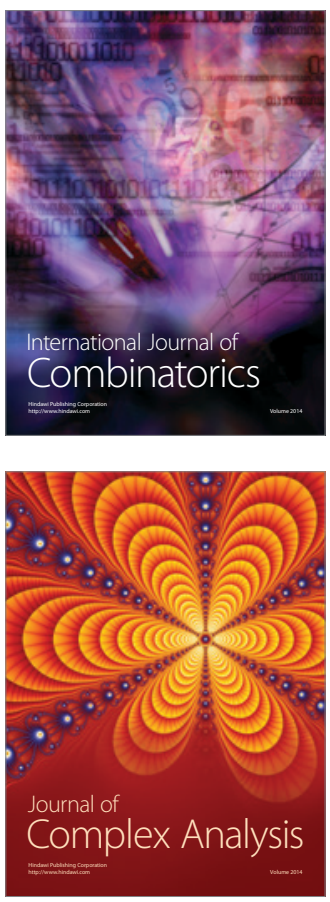

International Journal of

Mathematics and

Mathematical

Sciences
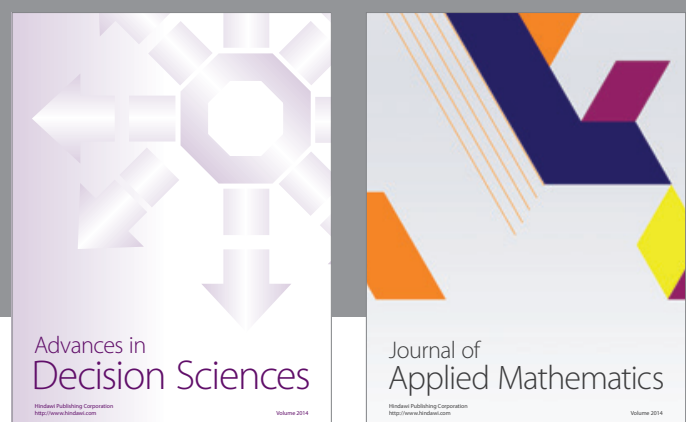

Journal of

Applied Mathematics
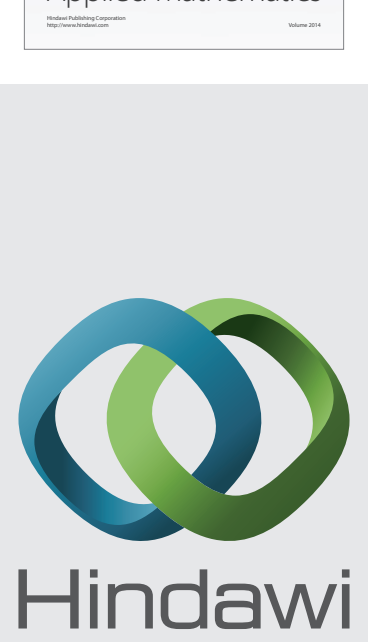

Submit your manuscripts at http://www.hindawi.com
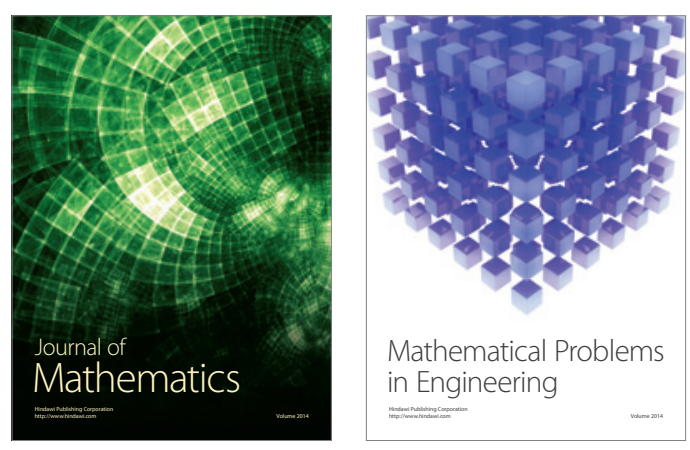

Mathematical Problems in Engineering
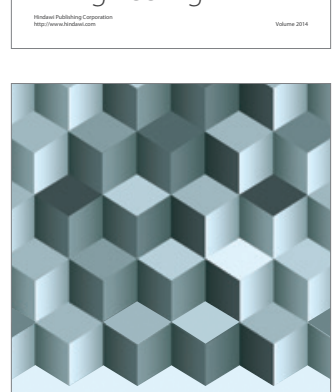

Journal of

Function Spaces
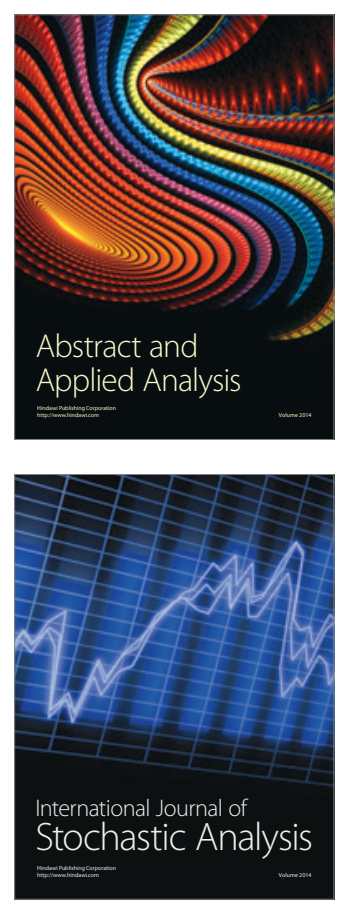

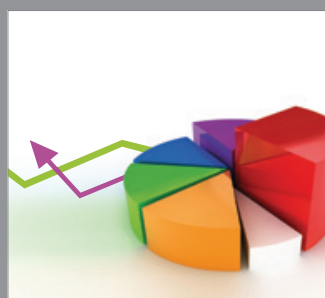

ournal of

Probability and Statistics

Promensencen
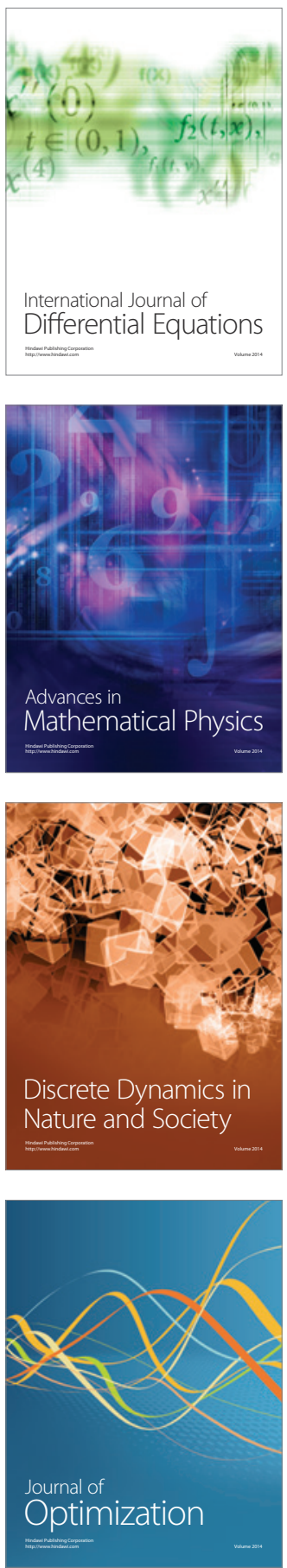